\title{
Bivalent HPV16/18 Therapeutic Cervical Cancer Vaccine
}

National Cancer Institute

\section{Source}

National Cancer Institute. Bivalent HPV16/18 Therapeutic Cervical Cancer Vaccine. NCI

Thesaurus. Code C112207.

\begin{abstract}
A bivalent human papillomavirus (HPV) therapeutic vaccine containing recombinant inactivated adenylate cyclase (CyaA) from Bordetella pertussis carrying a sequence encoding the E7 antigen of both HPV16 and 18, with potential immunostimulatory and antiviral properties. Upon administration of bivalent HPV16/18 therapeutic cervical cancer vaccine, the expressed proteins may activate cell-mediated immunity and induce both cytotoxic CD8+ T cells and CD4+ helper T cells against the target antigens HPV16-E7 and HPV18-E7, which leads to HPV viral clearance. Adenylate cyclase is a virulence factor of Bordetella pertussis. Its ability to bind to CD11 b-expressing dendritic cells and deliver antigens directly to the cytosol allows the activation and induction of T-cell immunity. CyaA may also induce a B cell response.
\end{abstract}

